INVESTIGACIÓN/RESEARCH

\title{
COMUNICACIÓN DE CRISIS: COMPILACIÓN Y REVISIÓN DE TEORÍAS Y TAXONOMÍAS PRÁCTICAS DESDE UNA PERSPECTIVA CUALITATIVA
}

Damián García Ponce1: Universidad de Málaga, España. damiangponce@gmail.com

Emilia Smolak-Lozano: Universidad de Málaga, España. emiliasmolak@gmail.com

\section{RESUMEN}

En la presente investigación exponemos, tras una revisión bibliográfica en profundidad las distintas teorías y modelos correspondientes a la Comunicación de Crisis; para ello realizamos una búsqueda y lectura cualitativa de libros y revistas especializadas e indizadas como Public Relations Quarterly, Public Relations Research, Journal of Public Relations y otras utilizando una serie de palabras clave y acotándolo a los resultados obtenidos en los últimos diez años, con referenciaciones, en la profundidad de la investigación, a algunas necesarias revisiones de textos anteriores. Posteriormente atacamos la biliografía resultante y la organizamos para obtener un marco completo e integrador del panorama teórico y práctico de la Comunicación de Crisis y cómo se ordena, clasifica y taxonomiza para, en las conclusiones, obtener un marco útil y práctico de uso de estos modelos además de reflexionar sobre la evolución sufrida en este campo de la Comunicación.

PALABRAS CLAVE: Comunicación de Crisis - Comunicación Organizacional - Crisis - Taxonomías - Evolución en Comunicación de Crisis - Gestión de Riesgos potenciales

\footnotetext{
${ }^{1}$ Autor Correspondiente

Damián García Ponce. Licenciado en Publicidad y Relaciones Públicas, Master en Gestión eficaz e innovación en comunicación y doctorando de Comunicación Estratégica en la Universidad de Málaga.

Correo: damiangponce@gmail.com
} 


\title{
CRISIS COMMUNICATION: COMPILATION AND REVIEW OF THEORIES AND PRACTIC TAXONOMIES IN A QUALITATIVE PERSPECTIVE
}

\begin{abstract}
This research paper aims to present the exhaustive review that was carried out on various Crisis Communication theories and models coming from various authors and schools by researching the bibliographic sphere that includes books and specialized indexed journals, such as: Public Relations Quarterly, Public Relations Research, Journal of Public Relations and others. The series of keywords were used and the results obtained were matched and filled along covering the perod of the last ten years, referencing, throughout the research, some necessary revisions of earlier texts. Then the resulting literature was analized and organized in order to obtain a complete and comprehensive framework of both theoretical and practical overview on Crisis Communication. Finally, the obtained research material has been sorted, graded and taxonomized, therefore, as the conclusion, the useful framework has been elaborated that is focused on the practical use of Crisis Communication models, reflecting the so far evolution of this communication area.
\end{abstract}

KEYWORDS: Crisis communication - organizational communication - crisis Taxonomies - Crisis Communication Evolution - Issues management.

\section{INTRODUCCIÓN}

Pretendemos en la presente investigación presentar una revisión en profundidad de las teorías, taxonomías y diversas clasificaciones realizadas por autores especializados y presentadas tanto en libros como en revistas, de la Comunicación de Crisis.

Con esta compilación, nuestra intención es ofrecer un marco integrador de uso para investigadores y profesionales así como una referenciación oportuna para profundizar en los diversos aspectos y tipos de teorías surgidas alrededor de este campo en los últimos años (aproximadamente un margen de una década).

La Comunicación de Crisis ha experimentado ciertos cambios desde la inclusión en el discurso de Internet como medio difusor de la palabra y la comunicación, debido, entre otras cosas a algunas de sus características, tales como: la inmediatez, la ubicuidad y la disponiblidad (Landau 2011: 58).

La evolución de los sistemas comunicativos de unidireccionales a bidireccionales, y de éstos a los sistemas complejos no lineales, que han dado una vuelta de tuerca a la expresión one to many, ha hecho que la Comunicación de Crisis también se adapte a estas circunstancias y utilice las mismas herramientas y el mismo lenguaje y código 
que los usuarios de Redes como Internet, si se pretende que la labor comunicativa tenga efecto.

Ya no basta con la publicación de una nota de prensa y un comunicado de prensa. Ya no son solo las grandes firmas y columnistas las opiniones influyentes. Ahora un joven con un blog puede convertirse en un tecnoinfluenciador (Burston-Masteller, 2005), o las Redes Sociales pueden trocarse en campos de batalla de la opinión y la comunicación de las organizaciones.

La compilación de las teorías y taxonomías de la Comunicación de Crisis puede suponer una ayuda, una "caja de herramientas" comunicacional con la que entender, analizar y reaccionar (con esperanzas en la proacción) este nuevo mundo, más veloz, rápido y volátil.

\section{METODOLOGÍA}

En la presente investigación realizamos una revisión en profundidad de la literatura existente en el campo de la Comunicación de Crisis, tanto en libros como en revistas especializadas en el campo de la comunicación, con el requisito de que estuvieran indizadas, tales como Public Relations Review, Public Relations Quarterly, Journal of Public Relations, etc. Para acotar la búsqueda situamos un marco temporal de resultados en un lapso de diez años (contando desde 2012), en el caso de las revistas, no siendo acotado el caso de los libros, debido a su menor periodicidad intrínseca.

Los resultados obtenidos en el caso de las revistas sugirieron además, la necesidad de puntuales revisiones de investigaciones y comunicaciones anteriores a ese marco de diez años para poder contextualizar ciertas teorías, acudiendo, de forma concienzuda y profesional, a las fuentes primeras de publicación de ciertas teorías, como es el caso de la Teoría Situacional de la Comunicación de Crisis, cuyas bases se encuentran en Commbs (1995). Este ejemplo hizo que nos remontáramos hasta dicha publicación para encontrar la base de ulteriores modelos construidos en torno a esos trabajos primeros de algunos autores determinados.

Los resultados fueron organizados y examinados en función de diversos parámetros no priorizados sino agrupados en función de temporalidad, efectos, tipologías agrupadas por diversos autores y teorías.

Tras esta compilación de datos siguió la revisión cualitativa de los mismos y la extracción de unas conclusiones meditadas sobre el conjunto de datos observados.

\section{ANÁLISIS Y DISCUSIÓN}

Encontramos varios eslabones o divisiones en la Comunicación de Crisis en función de su elemento básico y desencadenante, o sea, la Crisis. Numerosos autores han fundamentado la teoría de este campo, en ocasiones confrontándola con la práctica y nos han permitido observar las tipologías y características derivadas de esta observación y catalogación. Muchas de esas divisiones han sido realizadas, matizadas, 
reformuladas y complementadas por autores posteriores. Vamos a atender en este artículo a las bases

Podemos situar las siguientes divisiones:

\subsection{Fases de las Crisis}

Independientemente de la definición a la que nos ciñamos, la Comunicación de Crisis identifica tres estadios en la evolución de las Crisis:

- Fase de Pre-crisis: En esta etapa, la crisis empieza a dar señales de vida y la organización empieza ya a prever y detectar la existencia de un posible problema futuro.

- Fase de Crisis: En esta etapa la crisis ya ha salido a la luz pública a través de los medios de comunicación.

- Fase de Post-crisis: Entendida como la fase de vuelta a la normalidad. A estas alturas del conflicto, la organización ha superado el punto álgido de la crisis y se enfrenta ahora a una etapa de recuperación. (González Herrero 1998)

Otras teorías afirman que el ciclo de vida de una crisis está medido desde un punto de vista lineal, mientras que el mundo que nos rodea no muestra ese comportamiento, sino una alta variabilidad e imprevisibilidad cíclica o presuntamente cíclica. Quienes más se acercan a esta concepción son aquellos que han aplicado a la comunicación de crisis la Teoría del Caos. Ésta teoría que se adentra en las matemáticas no lineales, ahondando en la búsqueda de un patrón entre el Caos aparente y la autoorganización presente, por ejemplo en las muestras matemáticas, gráficas y en la misma naturaleza de los patrones fractales y atractores. A este respecto, los autores (Speakman y Sharplay, 2012; Seeger 2002) aplican el modelo del Caos a la Comunicación de Crisis, como Jaques (2007) que defiende una estructura cíclica en lugar de lineal (como veremos más adelante).

\subsection{Tipologías}

Las crisis se presentan sin avisar, son un invitado contra el que podemos estar en mayor o menor medida prevenidos y provocan desestabilización en el seno de nuestra empresa. Las crisis pueden provocar una respuesta adversa de nuestro entorno, pero una buena gestión de comunicación no sólo puede paliar sus efectos, sino que, además, con la adecuada gestión del plan puede llegar a convertirse en un futuro beneficio para la empresa, reforzando su imagen pública y su posición con respecto a la competencia.

Una de las taxonomías más significativas que encontramos es la que hace Castillo Esparcia (2010: 207) recogiendo las observaciones de distintos autores para componer una visión global de dicha taxonomía según autores: 
Comunicación de Crisis - Comunicación Organizacional - Crisis - Taxonomías Evolución en Comunicación de Crisis - Gestión de Riesgos potenciales

\begin{tabular}{|c|c|}
\hline \multicolumn{2}{|c|}{$\begin{array}{c}\text { Tabla 1. Taxonomía de las Crisis } \\
\text { Taxonomía de las crisis }\end{array}$} \\
\hline AUTOR & CLASIFICACIÓN \\
\hline $\begin{array}{l}\text { Institute for Crisis } \\
\text { Management }\end{array}$ & $\begin{array}{l}\text { - Operativas } \\
\text { - De gestión }\end{array}$ \\
\hline Gottschalk & $\begin{array}{l}\text { - Financieras } \\
\text { - De imagen } \\
\text { - Agentes externos }\end{array}$ \\
\hline $\begin{array}{l}\text { Berge } \\
\text { Mayers y Holusha }\end{array}$ & $\begin{array}{l}\text { - Tantos tipos de crisis como situaciones } \\
\text { problemáticas }\end{array}$ \\
\hline Berge & $\begin{array}{l}\text { - Crónicas (no emergencias) } \\
\text { - Agudas (emergencia) }\end{array}$ \\
\hline Reihardt & $\begin{array}{l}\text { - Inmediatas (emergencia) } \\
\text { - En desarrollo ( no emergencia) }\end{array}$ \\
\hline $\begin{array}{l}\text { Mitroff, Pauchant } \\
\text { Shrivastava }\end{array}$ & $\begin{array}{l}\text { - Técnico/Externa } \\
\text { - Económico/Externa } \\
\text { - Personal-social organizativa/Interna } \\
\text { - Personal-social organizativa/Externa }\end{array}$ \\
\hline Pauchant y Mitroff & $\begin{array}{l}\text { - Ataques económicos/Externos } \\
\text { - Ataques sobre la información/Externos } \\
\text { - Fallos } \\
\text { - Megafallos } \\
\text { - Enfermedades laborales } \\
\text { - Crisis psicológicas }\end{array}$ \\
\hline González Herrero & $\begin{array}{l}\text { - Evitables } \\
\text { - No evitables (accidentes u operativas) }\end{array}$ \\
\hline
\end{tabular}

Fuente: Castillo Esparcia, 2010:207

En cuanto las tipologías de crisis podemos distinguir varias, atendiendo a su origen y tipos. A continuación veremos estas clasificaciones.

Múltiples son los orígenes, y éstos determinarán el tipo de crisis al que nos enfrentamos y los instrumentos comunicativos de que habremos de disponer; asimismo activaremos el plan adecuado y de no haberlo, usaremos los parámetros básicos de un plan de crisis, matizándolos en función de las necesidades debido al tipo de crisis desatado.

\subsubsection{Orígenes de las crisis}

Según Piñuel y Westphalen (1993:87-100) los orígenes de las crisis pueden ser:

\subsubsection{Objetivos / subjetivos:}

a) Objetivos: Acontecimientos externos.

b) Subjetivos: Aquellos provocados por personas relacionadas directa o indirectamente con la empresa, ya se trate de ex-empleados, periodistas, miembros de otras empresas enfrentados, etc.

\subsubsection{Técnicos / políticos:}


a) Técnicos: relacionados con la cadena o algunos procesos de fabricación del producto.

b) Políticos: referentes a la política de la empresa.

\subsubsection{Exógenos / Endógenos:}

a) Exógenos: subida de los costes de las materias primas.

b) Endógenos: aquellos que hacen primer blanco en el interior de la empresa. En su cohesión interna en primer lugar, descontento entre los trabajadores, etc. y así se extiende al exterior. No olvidemos que si no hay una buena comunicación interna, ésta, al estar ligada a la externa, repercutirá negativamente creando un foco de Crisis.

\subsubsection{Tipos de Crisis}

En cuanto a los tipos de crisis existe obviamente una cierta variedad con diversas causas y efectos.

Según Coombs Existen cuatro tipos de crisis, que recogió en su Situational Crisis Communication Theory, la Teoría Situacional de la Comunicación de Crisis, basada en la Teoría de la Atribución, esto es, en un marco de agentes externos, usuarios, afectados y no afectados, suelen dejarse guiar por directrices emocionales a la hora de atribuir la responsabilidad de una situación o crisis (1995: 455-456):

1. Faux Pas: o metedura de pata Iniciadas por agentes externos que pretenden enfrentarse a una organización, aprovechadno un error cometido por ésta o por un miembro de ella (manifestaciones, boicots...)

2. Accidentes: suceso inintencionado acaecido durante el normal desarrollo de sus operaciones organizacionales. Incluye en este aspecto actos naturales y de errores de inducción humana.

3. Transgresiones: acciones intencionales tomadas por una organización a sabiendas del riesgo y la posibilidad de herir.

4. Terrorismo: Acciones intencionales llevadas a cabo por actores con la finalidad de herir a la organización directa o indirectamente.

Ligado a este aspecto, y como elementos que matizan las actuaciones con respecto a las crisis, así como las posibles reacciones del público, Coombs establece en la asignación de atribuciones de responsabilidad los siguientes puntos (1995: 454, 457461):

1. Veracidad de las pruebas: refiriéndose a las pruebas de si el evento-crisis ha ocurrido y si este es verdadero, falso o ambiguo.

2. Daño: clasificado en severo o menor. Severo es el daño que incluye heridas graves, muerte, o daños masivos a la propiedad, mientras que menor es el daño que incluye heridas leves o daños a la propiedad. Los públicos en este punto se dividen entre víctimas y no víctimas.

3. Interpretación de la historia: positiva o negativa. Una historial de crisis similares hace que la causa de la aparición de las crisis se vea como permanente o 
estacionaria, mientras que una interpretación positiva de la historia hace que la aparición de la crisis sea esporádica.

Otro autor, Lerbinger las clasifica en siete tipos distintos las crisis que pueden darse (1997) por su origen de surgimiento:

1. Crisis naturales.

2. Crisis tecnológicas.

3. Crisis de confrontación. (Aquellas deliberadamente provocadas por individuos y grupos descontentos que luchan contra empresas, gobiernos y varios grupos de interés, en casos extremos buscando cambios radicales en el sistema).

4. Crisis de Malevolencia. (Cuando opositores o malhechores [sic... miscreant persons] usan medios criminales $\mathrm{u}$ otras tácticas extremas para el propósito de expresar hostilidad hacia, o búsqueda de beneficios de una compañía, país o sistema económico, quizás con el objetivo de desestabilizarlo o destruirlo (1997:144).

5. Crisis de gestión de valores sesgados. (Entran en el ámbito de las crisis derivadas de mala gestión directiva (management failure), junto con las de decepción y las de mala conducta (1997:186)

6. Crisis de decepción. (Son aquellas en las que la dirección oculta o falsifica información sobre la organización o los productos en sus tratos con los consumidores 1997:217)

7. Crisis de gestión de mala conducta. (En las que se roza deliberadamente la amoralidad e ilegalidad en sus conductas, (1997:242) como la corrupción, sobornos, etc).

Vemos la clasificación de Villafañe (1998) en función de sus características de repercusiones en los distintos ámbitos de la organización:

1. Catástrofes: Entraña un serio peligro para la empresas sobre todo por las consecuencias externas, de tipo social, ecológicas, y por su gran impacto en el público externo.

2. Fallos funcionales graves: fallos en la cadena de producción.

3. Crisis de honorabilidad: Corrupción en cualquiera de sus sentidos en los empleados o ejecutivos, sobornos, extorsión, espionaje industrial.

4. Amenazas económico-financieras: fracaso en los mercados de valores, fallos en la dirección, OPA hostil.

5. Crisis internas: conflictos laborales, baja competitividad, cambios traumáticos en la dirección.

Por otro lado, Mitroff $(2001: 34,35)$ clasifica de la siguiente manera la tipología de las Crisis Mayores. Destaca este autor, que las Crisis Mayores ocurren no sólo por lo que una organización sabe, anticipa y prepara planes, sino que ocurren muchas veces por aquello que no sabe, y no anticipa:

1. Económicas. (Huelgas, conflictividad laboral, escasez laboral, crack de los mercados...)

2. Informacionales. (Pérdida de información propia confidencial, información falsa, alteración de registros computerizados...) 
3. Físicas (pérdida de plantas e instalaciones claves)

4. De Recursos Humanos. (Pérdida de ejecutivos clave, pérdida de personal clave, alto absentismo, vandalismo y accidentes, violencia en el lugar de trabajo...)

5. Reputacional. (Calumnias, rumores, bromas pesadas, daños a la reputación corporativa, manipulación de los logos corporativos...)

6. De Actos Psicopáticos. (Manipulación de los productos, secuestros, toma de rehenes, terrorismo...).

7. Desastres naturales. (Terremotos, Tsunamis, explosiones, tifones, huracanes...).

\subsection{Ciclos de la Crisis}

La duración también es determinante, conociendo el ciclo de vida y estadio en que se encuentra, deberemos adaptar el plan, según el nivel de sorpresa con que nos haya aparecido (Del pulgar Rodriguez 1999: 130). Hay que tener por cierto que los efectos de una crisis no acaban en cuanto el momento de alarma ha concluido, sino que tiene unas repercusiones de variada índole en función de los efectos producidos en el entorno.

Podemos distinguir cuatro etapas en las crisis, según Piñuel y Westphalen (1993):

1. Fase Preliminar: Cuando se empiezan a advertir señales que nos pueden levar a la crisis. Lo deseable es que, prevenidos por éstos síntomas, se empiecen a aplicar decisiones correctas para subsanar cualquier posible acontecimiento que desencadene la crisis. Villafañe (1998) añade que "la mejor prevención es una Imagen Positiva".

2. Fase Aguda: aumenta la presión de los medios de comunicación, dando cobertura máxima al acontecimiento; la especulación puede llegar a distorsionar la información con consecuencias inesperadas y peligrosas para la Imagen de la empresa si alcanza demasiada cobertura, pues no hemos de olvidar que los rumores se extienden muy rápido y generalmente son se índole negativo.

3. Fase Crónica: Consecuencias a varios niveles derivados de la crisis: intervenciones externas (investigaciones), apertura de negociaciones, consecuencias jurídicas...

4. Fase Postraumática: Momento de reflexión y extracción de balances. Se toman las decisiones derivadas del efecto acción-reacción, tales como refuerzo de las medidas de seguridad, reestructuración interna.

\subsection{Teoría de la Atribución de Responsabilidad}

La teoría de la Atribución de Responsabilidad de las Crisis, con respecto a su surgimiento y de la responsabilidad con respecto a esta, fue enunciada igualmente por Coombs y Holladay (2002) en los que introducían el término no ya de culpabilidad sino de Atribución de la Responsabilidad con respecto al desarrollo de una crisis.

La clasificación es la siguiente:

1. Crisis con Victimas: Crisis de Mínima Responsabilidad Atribuida

- Desastres naturales 
- Rumores

- Violencia en el lugar de trabajo

- Manipulación de productos/malevolencia

2. Crisis Accidentales; Baja responsabilidad Atribuida

- Desafíos: públicos que señalan a la organización que está trabajando de manera inapropiada.

- Accidentes por errores técnicos: equipo o tecnología que fallan y ello causa un accidente industrial.

- Error técnico en un producto lesivo: equipo o tecnología que al fallar han causado que un producto sea defectuoso y potencialmente lesivo.

3. Crisis Previsibles: Fuerte responsabilidad por la crisis.

- Accidentes por errores humanos.

- Daños provocados por un producto debido a errores humanos.

- Error Organizacional: produce que los públicos/usuarios estén en peligro y violación de la ley.

Esta teoría adaptada a las crisis resultantes del trasiego comunicacional de la Red, proviene de las anteriores formulaciones de Jin y Liu (2010) con respecto a la utilización de los blogs como detonantes de crisis mediáticas en Internet.

Este modelo propuesto para la incorporación del llamado social media a los procesos de crisis, ya sea ésta desatada en el entorno digital o no, el modelo establece que existen dos partes (Jin, Austin y Liu, 2011: 346):

1. Cómo los recursos y la forma de la crisis informativa institucional afecta a las organizaciones (determinando las opciones de respuesta; como vemos hereda parte del "matching" de la Situation Crisis Communication Theory)

2. Las Estrategias recomendadas de respuesta a la crisis de Social Media.

Según los autores hay tres tipos de públicos:

1. Creadores influyentes de contenidos Social Media (ya sean individuos $u$ organizaciones.

2. Seguidores Social Media que son consumidores de la información producida por los Creadores, relativa a la crisis.

3. Los Social Media inactivos que consumen la información de los primeros de manera indirecta a través de medios offline como el boca oído de los segundos, incluyendo en esto a los medios de comunicación tradicional que son seguidores de los primeros y los segundos, a su vez.

Todo ello puesto en relación con un diagrama octogonal continente de los cinco tipo de factores que afectan cómo las organizaciones se comunican antes, durante y después de las crisis, a saber: origen de la crisis, tipo, infraestructura, estrategia del mensaje y forma del mensaje; factores éstos emergidos de Jin (2009:310-313), correspondiente a una entrevista compleja a un panel de cuarenta expertos periodistas dotándolo de una opinión experta sobre el terreno que redunda en la fiabilidad del modelo. 
En un sistema tan interrelacionado como es Internet, la fluctuación comunicativa podría determinar las estrategias a utilizar, siempre que el rastreo de la crisis y el "matching", heredero del sistema de Coombs (1995), se realice de forma adecuada.

\subsection{Teorías integradoras}

Sin embargo, Jaques (2007:150) nos propone un modelo distinto, integrador de la Gestión de Riesgos Potenciales y de la Gestión de Comunicación de Crisis (Crisis Communication Management o CCM), según la Teoría del Caos. Dicha teoría se fundamenta en la impredectibilidad factorial de los sistemas complejos y sus progresiones no lineales de interrelación entre individuos. Teoría que busca, entre otras cosas entender esta complejidad caótica y encontrar patrones en ellas, tales como los fractales y los atractores en los sistemas comportamentales. Jaques ya enuncia que los primeros intentos de aplicación de la Teoría del Caos a la Comunicación de Crisis provienen de Murphy (1996) y Seeger (2003), en los que trataron de unificar una teoría mediante la no linealidad. La intención de este autor es la de crear un modelo circular, no lineal, en el que se contemple la gestión de comunicación de crisis y la de riesgos potenciales como un todo cíclico subdividido, a su vez, en grupos.

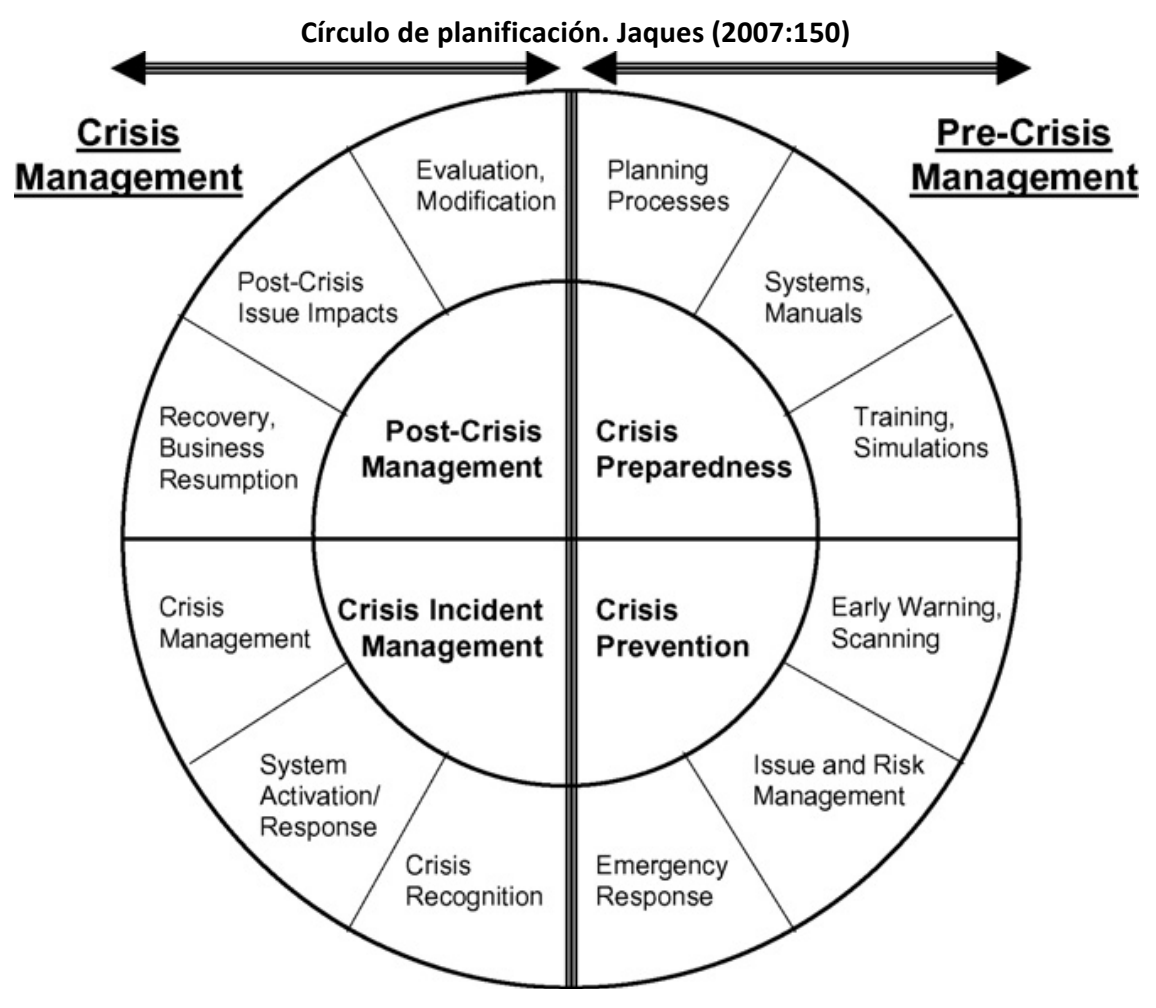

Podemos apreciar en este modelo que las divisiones corresponden a distintos modelos de la planificación y trabajo de gestión comunicativa. El círculo se encuentra dividido por cuatro radios de noventa grados en función de las distintas etapas:

1. La preparación de Crisis, subdividida a su vez en:
a. Procesos de Planificación
b. Elaboración de Sistemas y Manuales
c. Entrenamientos y Simulacros, 
2. Prevención de Crisis, constituida por

a. Alertas tempranas y monitorización del entorno

b. La Gestión de asuntos y riesgos potenciales

c. Respuestas de emergencia

3. Gestión de Crisis

a. Reconocimiento de la Crisis

b.Sistemas de activación / respuesta

c. Gestión de la Crisis

4. Gestión Post Crisis

a. Reanuación y recuperación de negocio

b. Análisis de Impacto Post-Crisis

c. Evaluación y Modificación

\section{CONCLUSIONES}

Tras esta extensa revisión bibliográfica las conclusiones del investigador centrado en el tema de la Comunicación de Crisis le llevan a observar las evoluciones de las clasificaciones diversas y las taxonomías aportadas por varios autores en sus respectivos trabajos. Partimos de las bases en los años 70 en los que se fundamentaba la Teoría de la Comunicación hasta la aplicación actual de teorías que permiten estudiar la interacción en sistemas complejos y realizar una adaptación de la Comunicación de Crisis a estos sistemas.

¿Por qué ha surgido esa necesidad de cambio de la linealidad? Debido, en gran parte, a las características de ese nuevo sistema de comunicación que es Internet, aunque no exclusivamente por ello. Las capacidades de este sistema que permite una fluctuación distinta de la información y de la comunicación hace que el comunicador deba integrar un modelo capaz de relacionarse en esos sistemas complejos que comportan un riesgo comunicacional. Ya no se trata de aplacar una crisis o adelantarse publicando una información para paliar un posible asunto (issue), sino que se trata de que esa información reciba suficiente eco y dispersión en los medios actuales para ser efectivo. Esa impredectibilidad de los sistemas es lo que da valor a una comunicación como esta, en la que se exponen los diversos tipos de Crisis hallados e investigado, en los que el investigador y el profesional pueden actuar, utilizar y complementar con sus propios hallazgos y observaciones para llevar a cabo esa labor esencial en la comunicación de las organizaciones.

Al suponer las crisis un periodo de desestabilización de la organización contar con un material que permita acotar el campo de actuación y suponga el primer paso para crear un marco situacional, los modelos aquí presentados cobran entonces su valor efectivo y de actuación, pues conocer a lo que nos estamos enfrentando es el primer paso para solucionarlo.

Los modelos integradores no secuenciales sino cíclicos presentan ventajas con respecto a los habituales fundamentados en el tiempo de vida de las crisis (precrisis, crisis, postcrisis), como es la integración de la Gestión de Riesgos Potenciales en el propio 
ciclo. A fin de cuentas la aparición de una Crisis no es más que el fracaso de la Gestión de Riesgos Potenciales y deriva directamente en la creación del asunto conflictivo y su desarrollo. Por lo tanto un modelo que permita desde la gestión de postcrisis, como afirma Jaques (2007) la aplicación de la gestión preventiva mejora las capacidades del equipo comunicativo a la hora de enfrentar las crisis, pues no todas ellas son evitables. Estamos sin duda a las puertas de la fundamentación de modelos y teorías que acerquen la comunicación de crisis a los mundos virtuales-digitales, entre otros, de Internet, donde las leyes y las formas cambian de continuo. En este espacio mutante se darán a conocer en mayor profundidad complementariedades a la Comunicación de Crisis y la Gestión de Riesgos Potenciales, como pueden ser los modelos de Paracrisis (Coombs y Holladay, 2012).

Otro aspecto importante que hemos anotado es que las teorías aquí reflejadas, las taxonomías y modelos han sido todos extraídos de los textos observados, con lo que se deduce que gran parte de los textos utilizan las teorías aquí expuestas o reflexionan y usan algunas partes de éstas en aspectos muy concretos, lo que nos da como resultado una diferencia apreciable entre la cantidad de bibliografía con respecto a la de teorías y modelos aquí observados.

La mayoría de las teorías, asimismo, que se utilizan para la aplicación a los nuevos entornos comunicativos proceden de la literatura especializada y es una aplicación, como hemos dicho, en lugar de ser teorías o modelos surgidos específicamente para estos nuevos entornos.

Por ellos una de las conclusiones deriva en la viabilidad del surgimiento de una Teoría aplicada desde la observación pura del entorno virtual.

\section{REFERENCIAS}

Arpan, L. M, Pompper, D. (2003). “Stormy wheather: tesing 'stealing thunder' as a crisis communication strategy to improve communication flow between organizations and journalists". En Public Relations Review. núm. 29. (pp. 291-308).

Arpan, L. M; Roskos-Ewoldsen, D. R. (2005) "Stealing thunder: analysis of the effects of proactive disclosure of crisis information". En Public Relations Review. núm. 31. (pp. 425-433).

Avery, E. J.; Larisacy, R. W.; Kim, S; Hocke, T. (2010) “A quantitative review of crisis communication research in public relations from 1991 to 2009". En Public Relations Review. núm 36. (pp 190-192).

Bartos, O \& Wehr, P. (2002): Using Conflict Theory. Cambridge University Press, New York.

Bridgeman, $\mathrm{R}$ en Anthonissen, P. Crisis Communication: Practical PR Strategies for Reputation Management and Company Survival. London, GBR: Kogan Page Ltd., 2008. (pp. 177-195). 
Comunicación de Crisis - Comunicación Organizacional - Crisis - Taxonomías Evolución en Comunicación de Crisis - Gestión de Riesgos potenciales

Brown, K. A. \& White, C. L. (2010): “Organization-Public Relationships and Crisis Response Strategies: Impact on Attribution of Responsibility". En Journal of Public Relations Research, 23:1, (pp. 75-92).

Castillo Esparcia, A. (coord.) (2005): Comunicación organizacional. Teorías y estudios, Clave Aynadamar. Málaga.

Castillo Esparcia, A. (2010): Introducción a las relaciones públicas. Instituto de Investigación en Relaciones Públicas. España.

Chase, W. H. (1984). Issue management - origins of the future. Stamford, CT: Issue Action Publications.

Christen, C. T. (2005). “The restructuring and reengieneering of AT\&T: Analysis of a public relations crisis using organizational theory". En Public Relations Review. núm. 31. (pp. 239-251).

Claeys, A, Caugerghe, V. (2012) "Crisis response and crisis timing strategies, two sides of the same coin". En Public Relations Review. núm. 38. (pp. 83-88).

Coombs, W. T. (1995). "Choosing the right words: The development of guidelines for the selection of the "appropriate" crisis response strategies" en Management Communication Quarterly, 8: (pp. 447-476).

Coombs, W. T., \& Holladay, S. J. (2002). “Helping crisis managers protect reputational assets: Initial tests of the situational crisis communication theory". En Management Communication Quarterly, 16: (pp. 165-186).

Coombs, W.T. (2004). "Impact of Past Crises on Current Crisis Communication: Insights From Situational Crisis Communication Theory". En Journal of Business Communications. Núm. 41 (pp. 265-289).

Coombs, W.T. (2007) Crisis management and communications. Institute of Public Relations. http://www.instituteforpr.org/topics/crisis-management-andcommunications/ (consultado 1 de noviembre 2012).

Coombs, W.T.; Holladay, J. S. (2012) “The paracrisis: the challenges creates by publicly managing crisis prevention". En Public Relations Review. num: 38. (pp. 408-435).

Coombs. W. T. (2007). “Attribution Theory as a guide for post-crisis communication research". En Public Relations Review. núm 33 (pp 135-139).

Craig, M; Olaniran, B; Scholl, J. C.; Williams, D. E. (2006) “Crisis communication in public arenas". En Public Relations Review. núm. 32. (pp. 171-173).

Dinardo, A. (2002) "The Internet as a crisis management tool: a critique of banking sites during Y2K”. En Public Relations Review. núm. 28. (pp. 367-378). 
Dotty, O. (2005): The hostile media effect: a state of the art review. Johns Hopkins University. Washington D.C.

Fita Trías, J. (1999): Comunicación en programas de Crisis. Gestión 2000. Barcelona.

González-Herrero, A. (1998): Márketing Preventivo: la comunicación de crisis en la empresa. Bosch Comunicación. Barcelona.

González-Herrero, A y Smith, S. (2008) "Crisis communications management on the Web: How Internet-based technologies are changing the way public relations professionals handle business crises". Journal of Contingencies and Crisis Management. Vol. 16. Núm. 3. (pp. 143-153).

Halpern, D. (2008): “Crisis 2.0: La necesidad de un modelo integrado de comunicación". En Cuadernos de información. No22, (pp 48-57).

Heath, R. L. (1997). Strategic issues management: Organizations and public policy challenges. Thousand Oaks, CA: Sage.

Heath, R. L. (2002). “Issues management: Its past, present and future” en Journal of Public Affairs, 2(4), (pp. 209-214).

Huang, S. (2003): “Internet Based-Crises” en Razón y Palabra, Abril-Mayo.

Huang, Y (2006) “Crisis Situations, Communications Strategies, and Media Coverage: A multicase studi revisiting the Communicative Response Model". En Communications Research. Volumen: 33, Núm. 3. (pp. 180-205).

Huang, Y. (2008): "Trust and Relational Commitment in Corporate Crises: The Effects of Crisis Communicative Strategy and Form of Crisis Response" en Journal of Public Relations Research, 20:3, (pp. 297-327).

Jaques, T. (2009a). “Issue and crisis management: Quicksand in the definitional landscape" en Public Relations Review (pp. 280-286).

Jaques, T. (2009b) “Issue Management as a post-crisis discipline: Identifying and responding to issue impacts beyond the crisis". En Journal of Public Affairs, 9(1), (pp 35$44)$.

Jaques, T. (2007). “Issue management and crisis management: an integrated nonlinear, relational construct". En Public Relations Review. núm. 33. (pp. 147-157).

Jin, Y \& Liu B. F. (2010): “The Blog-Mediated Crisis Communication Model: Recommendations for Responding to Influential External Blogs". En Journal of Public Relations Research, 22:4, (pp. 429-455) 
Comunicación de Crisis - Comunicación Organizacional - Crisis - Taxonomías Evolución en Comunicación de Crisis - Gestión de Riesgos potenciales

Jin, Y. Pang, A. y Cameron, G. T. (2012): “Toward a Publics-Driven,

Emotion-Based Conceptualization in Crisis Communication: Unearthing Dominant Emotions in Multi-Staged Testing of the Integrated Crisis Mapping (ICM) Model". En Journal of Public Relations Research, 24:3, (pp. 266-298)

Kersten, A.(2005) "Crisis as usual: Organizational dysfunction and public relations". En Public Relations Review. num. 31. (pp. 544-549).

Lerbinger, O. (1997): The Crisis Manager. Facing risk and responsability. Lawrence Erlbaum Associates Publishers. New Jersey.

Liu, B. F., Austin, L., Jin, Y. (2011) “How publics respond to crisis communications strategies: The interplay of information form and source". En Public Relations Review. núm. 37 (pp 345-353).

Liu, B. F. (2010). “Distinguishing how elite newspapers and A-list blogs cover crises: Insights for managing crises online”. En Public Relations Review. núm. 36. (pp 28-34).

Marra, F. J. (1998) "Crisis Communication Plans: Poor Predictors of Excellent Crisis Public Relations". En Public Relations Review. 24(4). (pp. 461-474).

Matilla Serrano, K. (2010): “Issues Management. Más allá de la Gestión de Conflictos Potenciales". aDResearchESIC. Segundo Semestre, Julio-Diciembre 2010. (pp 40-57).

Mitroff, I. (2001): Managing crises before they happen. AMACOM. New York.

Mitroff, I. (2005): “Why some companies emerge stronger and better from crisis: 7 essential lessons". En American Management Association. New York.

Murphy, P. (1996). "Chaos theory as a model for managing issues and crisis". En Public Relations Review, 22(2) (pp. 95-113).

Regester, M. Larkin, J. (2005). Risk Issues and Crisis Management. A casebook of Best Practice. PR in Practice Series. CIPR. Londres.

RATZAN, S. C. (2005): “Communicating Risk: From Crisis to Calm”. En Journal of Health Communication: International Perspectives, 10:2, (pp. 103-104).

Seeger, M. W. (2002). "Chaos and crisis: Propositions for a general theory of crisis communication". En Public Relations Review, 28(4), (pp. 329-337).

Seeger, M. W. (2006): “Best Practices in Crisis Communication: An Expert Panel Process". En Journal of Applied Communication Research, 34:3, (pp. 232-244).

Speakman, M.\& Sharpley, R. (2012) "A chaos theory perspective on destination crisis management: Evidence from Mexico". En Journal of Destination Marketing $\mathcal{E}$ Management (pp 1-11). 
Stavem Kyhn, H. (2008). Situations crisis communication theory: its use in a complex crisis with Scandinavian Airlines Grounding of Dash 8-q400 airplanes. Aarhus School of Business. Aarhus Universitet. Denmark.

Sweetser, K. D.; Metzgar, E. (2007). “Communicating during crisis: use of blogs as a relationship management tool”. En Public Relations Review. núm. 33. (pp 340-342).

Taylor, M., Kent, M. L. (2007) “Taxonomy of mediated crisis responses”. En Public Relations Review. núm. 33. (pp. 140-146).

Taylor, M., Perry, D. C. (2005). “Diffusion of traditional and new media tactics in crisis communications". En Public Relations Review.. núm. 31 (pp. 209-217).

Tripier, B. (2007) "Crisis y oportunidad". Diario de Guayana. 12 de abril de 2007. Guayana.

Ulmer, R. R.; Seeger, M. W.; Sellnow, T. L. (2007) “Post-crisis communication and renewal: Expanding de parameters of post-crisis discourse". En Public Relations Review. núm 33. (pp. 130-134).

Venette, S. J. (2006): “Special Section Introduction: Best Practices in Risk and Crisis Communication". En Journal of Applied Communication Research, 34:3, (pp. 229-231).

Villafañe, J. (1993): Imagen positiva: gestión estratégica de la imagen de las empresas Pirámide, Madrid.

Villafañe, J. (1999) La gestión profesional de la imagen corporativa. Pirámide, Madrid.

Wilcox, D. C., Glen T., Xifra, J. (2001): Relaciones Públicas: estrategias y prácticas, Addison Wesley, Madrid.

Xifra, J. (2005): “Relaciones Públicas proactivas: el modelo de la gestión de conflictos potenciales", en CASTILLO ESPARCIA, A. (coord.), Comunicación organizacional. Teorías y estudios, Málaga: Clave Aynadamar, (pp. 215-243).

Xifra, J. (2005): Planificación estratégica de las Relaciones Públicas, Paidós, Barcelona.

\section{Damián García Ponce}

Licenciado en Publicidad y Relaciones Públicas, Master en Gestión Eficaz e Innovación y Comunicación, y doctorando en Direccion Estratégica en Comunicación, realiza una tesis doctoral sobre Hostilidad Comunicativa en Entornos Virtuales y realiza investigaciones sobre campos de comunicación, gamification, y coordina el Master en Comunicación 2.0. Social Media y Community Management en la Universidad de Málaga.

\section{Emilia Smolak-Lozano}


Licenciada en Sociología, Master en Gestión Eficaz e Innovación en Comunicación y Doctoranda de Ciencias de Comunicación de Universidad de Málaga. Socióloga de Internet, especializada en investigación de los medios de comunicación y en comunicación. 5 años de experiencia en varios proyectos de investigación de mercados y consumidores para las empresas. Además, está participando en varios proyectos de investigación científica en las Ciencias Sociales relacionados con comunicación 2.0. Actualmente, colabora con la empresa SmartNet Research and Solutions, haciendo investigaciones de comunicación 2.0 y audiencias en Facebook y Social Media mediante el software especializado Sotrender.com, de cuyo marketing está responsable en territorio español. Consultora de Social Media y evaluación en el mercado polaco. 